\title{
AN OPEN FRAMEWORK FOR RAPID PROTOTYPING OF SIGNAL PROCESSING APPLICATIONS
}

\author{
Maxime Pelcat ${ }^{1}$, Jonathan Piat ${ }^{1}$, Matthieu Wipliez ${ }^{1}$, Slaheddine Aridhi ${ }^{2}$, and Jean-François Nezan ${ }^{1}$ \\ (1) IETR/Image and Remote Sensing Group, CNRS UMR 6164/INSA Rennes \\ 20, avenue des Buttes de Coësmes, 35043 RENNES Cedex, France \\ email : \{mpelcat, jpiat, mwipliez, jnezan $\} @$ insa-rennes.fr \\ (2) Texas Instruments, HPMP Division, \\ 06271 Villeneuve Loubet, France \\ email : saridhi@ti.com
}

\begin{abstract}
Embedded real-time applications in communication systems have significant timing constraints, thus requiring multiple computation units. Manually exploring the potential parallelism of an application deployed on multi-core architectures is greatly time-consuming. This paper presents an open source Eclipse-based framework which aims to facilitate the exploration and development processes in this context. The framework includes a generic graph editor (Graphiti), a graph transformation library (SDF4J) and an automatic mapper/scheduler tool with simulation and code generation capabilities (PREESM). The input of the framework is composed of a scenario description and two graphs, one graph describes an algorithm and the second graph describes an architecture. The rapid prototyping results of a 3GPP Long Term Evolution (LTE) algorithm on a multi-core digital signal processor illustrate both the features and the capabilities of this framework.
\end{abstract}

Index Terms-Rapid prototyping, static scheduling, automatic code generation, generic graph editor, LTE

\section{INTRODUCTION}

The recent evolution of digital communication systems (voice, data and video) has been dramatic. Over the last two decades, low data-rate systems (such as dial-up modems, first and second generation cellular systems, 802.11 Wireless local area networks) have been replaced or augmented by systems capable of data rates of several Mbps, supporting multimedia applications (such as DSL, cable modems, 802.11b/a/g/n wireless local area networks, 3G, WiMax and ultra-wideband personal area networks).

As communication systems have evolved, the resulting increase in data rates has necessitated a higher system algorithmic complexity. A more complex system requires greater flexibility in order to function with different protocols in different environments. Additionally, there is an increased need for the system to support multiple interfaces and multicomponent devices. Consequently, this requires the optimization of device parameters over varying constraints such as performance, area and power. Achieving this device optimization requires a good understanding of the application complexity and the choice of an appropriate architecture to support this application.

An embedded system commonly contains several processor cores in addition to hardware coprocessors. The embedded system designer needs to distribute a set of signal processing functions onto a given hardware with predefined features. The functions are then executed as software code on target architecture ; this action will be called a deployment in this paper. A common approach to implement a parallel algorithm is is the creation of a program containing several synchronized threads in which execution is driven by the scheduler of an operating system. Such an implementation does not meet the hard timing constraints required by real-time applications and the memory consumption constraints required by embedded systems [1]. One-time manual scheduling developed for single-processor applications is also not suitable for multiprocessor architectures : manual data transfers and synchronizations quickly become very complex, leading to wasted time and potential deadlocks. Furthermore, the task of finding an optimal deployment of an algorithm mapped onto a multi-component architecture is not straightforward. When performed manually, the result is inevitably a sub-optimal solution. These issues raise the need for new methodologies, which allow the exploration of several solutions, to achieve a more optimal result.

Several features must be provided by a fast prototyping process : description of the system (hardware and software), automatic mapping/scheduling, simulation of the execution and automatic code generation. This paper draws on previously presented works [2][3][4] in order to generate a more complete rapid prototyping framework. This complete framework is composed of three complementary tools based on 
Eclipse [5] that provide a full environment for the rapid prototyping of real-time embedded systems : Parallel and Realtime Embedded Executives Scheduling Method (PREESM), Graphiti and Synchronous Data Flow for Java (SDF4J). This framework implements the methodology AlgorithmArchitecture Matching (AAM), which was previously called Algorithm-Architecture Adequation (AAA) [6]. The focus of this rapid prototyping activity is currently static code mapping/scheduling but dynamic extensions are planned for future generations of the tool.

From the graph descriptions of an algorithm and of an architecture, PREESM can find the right deployment, provide simulation information and generate a framework code for the processor cores [2]. These rapid prototyping tasks can be combined and parameterized in a workflow. In PREESM, a workflow is defined as an oriented graph representing the list of rapid prototyping tasks to execute on the input algorithm and architecture graphs in order to determine and simulate a given deployment. A rapid prototyping process in PREESM consists of a succession of transformations. These transformations are associated in a data-flow graph representing a workflow that can be edited in a Graphiti generic graph editor. The PREESM input graphs may also be edited using Graphiti. The PREESM algorithm models are handled by the SDF4J library. The framework can be extended by modifying the workflows or by connecting new plug-ins (for compilation, graph analyses, and so on).

In this paper, the differences between the proposed framework and related works are explained in Section 2. The framework structure is described in Section 3. Section 4 details the features of PREESM that can be combined by users in workflows. The use of the framework is illustrated by the deployment of a wireless communication algorithm from the 3rd Generation Partnership Project (3GPP) Long Term Evolution (LTE) standard in Section 5. Finally, conclusions are given in Section 6.

\section{STATE OF THE ART OF RAPID PROTOTYPING AND MULTI-CORE PROGRAMMING}

There exist numerous solutions to partition algorithms onto multi-core architectures. If the target architecture is homogeneous, several solutions exist which generate multi-core code from $\mathrm{C}$ with additional information (OpenMP [7], CILK [8]). In the case of heterogeneous architectures, languages such as OpenCL [9] and the Multicore Association Application Programming Interface (MCAPI [10]) define ways to express parallel properties of a code.However, they are not currently linked to efficient compilers and runtime environments. Moreover, compilers for such languages would have difficulty in extracting and solving the bottlenecks of the implementation that appear inherently in graph descriptions of the architecture and the algorithm.

The Poly-Mapper tool from PolyCore Software [11] of- fers functionalities similar to PREESM but, in contrast to PREESM, its mapping/scheduling is manual. Ptolemy II [12] is a simulation tool that supports many models of computation. However, it also has no automatic mapping and currently its code generation for embedded systems focuses on singlecore targets. Another family of frameworks existing for data flow based programming is based on CAL [13] language and it includes OpenDF [14]. OpenDF employs a more dynamic model than PREESM but its related code generation does not currently support multi-core embedded systems.

Closer to PREESM are the Model Integrated Computing (MIC [15]), the Open Tool Integration Environment (OTIE [16]), the Synchronous Distributed Executives (SynDEx [17]), the Dataflow Interchange Format (DIF [18]), and SDF for Free (SDF3 [19]). Both MIC and OTIE can not be accessed online. According to the literature, MIC focuses on the transformation between algorithm domain-specific models and metamodels while OTIE defines a single system description that can be used during the whole signal processing design cycle.

DIF is designed as an extensible repository of representation, analysis, transformation and scheduling of data-flow language. DIF is a Java library which allows the user to go from graph specification using the DIF language to $\mathrm{C}$ code generation. However, the hierarchical Synchronous Data Flow (SDF) model used in the SDF4J library and PREESM is not available in DIF.

SDF3 is an open source tool implementing some data-flow models and providing analysis, transformation, visualization, and manual scheduling as a $\mathrm{C}++$ library. SDF3 implements the Scenario Aware Data Flow (SADF [20]), and provides Multiprocessor System-on-Chip (MP-SoC) binding/scheduling algorithm to output MP-SoC configuration files.

SynDEx and PREESM are both based on the AAM methodology [6] but the tools do not provide the same features. SynDEx is not open source, has its own model of computation that does not support schedulability analysis and code generation is possible but not provided with the tool. Moreover, the architecture model of SynDEx is at a too high level to account for bus contentions and DMA used in modern chips (multi-core processors of MP-SoC) in the mapping/scheduling.

The features that differentiate PREESM from the related works and similar tools are :

- The tool is an open source and accessible online,

- The algorithm description is based on a single wellknown and predictable model of computation,

- The mapping and the scheduling are totally automatic,

- The functional code for heterogeneous multi-core embedded systems can be generated automatically, 
- The algorithm model provides a helpful hierarchical encapsulation thus simplifying the mapping/scheduling [3].

The PREESM framework structure is detailed in the next section.

\section{AN OPEN SOURCE ECLIPSE-BASED RAPID PROTOTYPING FRAMEWORK}

\subsection{The Framework Structure}

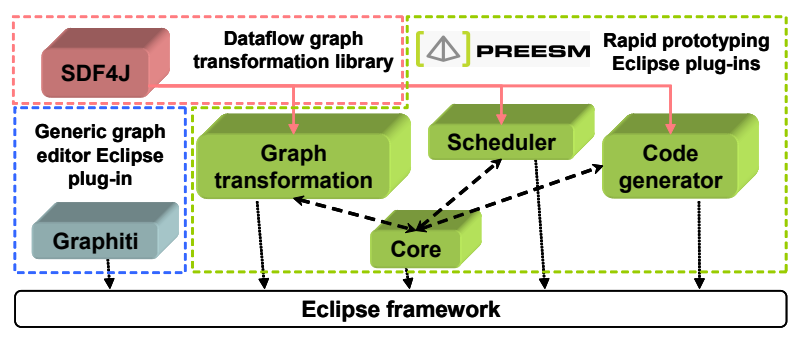

Fig. 1. An Eclipse-based Rapid Prototyping Framework

The framework structure is presented in Figure 1. It is composed of several tools to increase reusability in several contexts.

The first step of the process is to describe both the target algorithm and the target architecture graphs. A graphical editor reduces the development time required to create, modify and edit those graphs. The role of Graphiti [21] is to support the creation of algorithm and architecture graphs for the proposed framework. Graphiti can also be quickly configured to support any type of file formats used for generic graph descriptions.

The algorithm is currently described as a Synchronous Data Flow (SDF [22]) Graph. The SDF model is a good solution to describe algorithms with static behaviour. The SDF4J[23] is an open source library providing usual transformations of SDF graphs in the Java programming language. The extensive use of SDF and its derivatives in the programming model community led to the development of SDF4J as an external tool. Due to the greater specificity of the architecture description compared to the algorithm description, it was decided to perform the architecture transformation inside the PREESM plug-ins.

The PREESM project [24] involves the development of a tool that performs the rapid prototyping tasks. The PREESM tool uses the Graphiti tool and SDF4J library to design algorithm and architecture graphs and to generate their transformations. The PREESM core is an Eclipse plug-in that executes sequences of rapid prototyping tasks or workflows. The tasks of a workflow are delegated to PREESM plug-ins. There are currently three PREESM plug-ins : the graph transformation plug-in, the scheduler plug-in and the code-generation plug-in.
The three tools of the framework are detailed in the next sections.

\subsection{Graphiti : A Generic Graph Editor for Editing Ar- chitectures, Algorithms and Workflows}

Graphiti is an open-source plug-in for the Eclipse environment that provides a generic graph editor. It is written using the Graphical Editor Framework (GEF). The editor is generic in the sense that any type of graph may be represented and edited. Graphiti is used routinely with the following graph types and associated file formats : CAL networks [13, 25], a subset of IP-XACT [26], GraphML [27] and PREESM workflows [28].

\subsubsection{Overview of Graphiti}

A type of graph is registered within the editor by a configuration. A configuration is an XML (Extensible Markup Language [29]) file that describes :

1. The abstract syntax of the graph (types of vertices and edges, and attributes allowed for objects of each type),

2. The visual syntax of the graph (colors, shapes, etc.),

3. Transformations from the file format in which the graph is defined to Graphiti's XML file format $\mathcal{G}$, and viceversa (Figure 2).

Two kinds of input transformations are supported, from XML to XML and from text to XML (Figure 2). XML is transformed to XML with Extensible Stylesheet Language Transformation (XSLT [30]), and text is parsed to its Concrete Syntax Tree (CST) represented in XML according to a LL(k) grammar by the Grammatica [31] parser. Similarly, two kinds of output transformations are supported, from XML to XML and from XML to text.

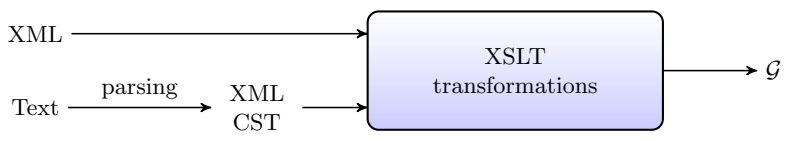

(a) reading an input file to $\mathcal{G}$

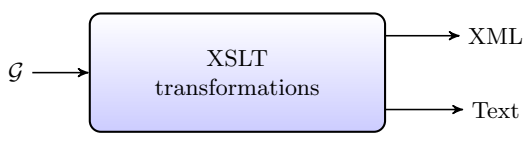

(b) writing $\mathcal{G}$ to an output file

Fig. 2. Input/output with Graphiti's XML format $\mathcal{G}$

Graphiti handles attributed graphs [32]. An attributed graph is defined as a directed multigraph $G=(V, E, \mu)$ with $V$ the set of vertices, $E$ the multiset of edges (there can be more than one edge between any two vertices). $\mu$ is a function $\mu:(\{G\} \cup V \cup E) \times A \mapsto U$ that associates instances 
with attributes from the attribute name set $A$ and values from $U$, the set of possible attribute values. A built-in type attribute is defined so that each instance $i \in\{G\} \cup V \cup E$ has a type $t=\mu(i$, type $)$, and only admits attributes from a set $A_{t} \subset A$ given by $A_{t}=\tau(t)$. Additionally, a type $t$ has a visual syntax $\sigma(t)$ that defines its color, shape and size.

To edit a graph, the user selects a file and the matching configuration is computed based on the file extension. The transformations defined in the configuration file are then applied to the input file and result in a graph defined in Graphiti's XML format $\mathcal{G}$ as shown in Figure 2. The editor uses the visual syntax defined by $\sigma$ in the configuration to draw the graph, vertices and edges. For each instance of type $t$ the user can edit the relevant attributes allowed by $\tau(t)$ as defined in the configuration. Saving a graph consists of writing the graph in $\mathcal{G}$, and transforming it back to the input file's native format.

\subsubsection{Editing a Configuration for a Graph Type}

To create a configuration for the graph represented in Figure 3, a node (a single type of vertex) must be defined. A node has an unique identifier called $i d$, and accepts a list of values initially equal to [0] (Figure 4). Additionally, ports need to be specified on the edges, so the configuration describes an edge Type element (Figure 5) that carries sourcePort and targetPort parameters to store an edge's source and target ports respectively, such as acc, in, and out in Figure 3.

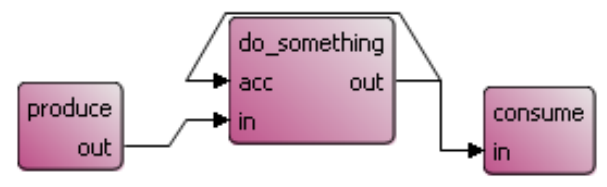

Fig. 3. A sample graph

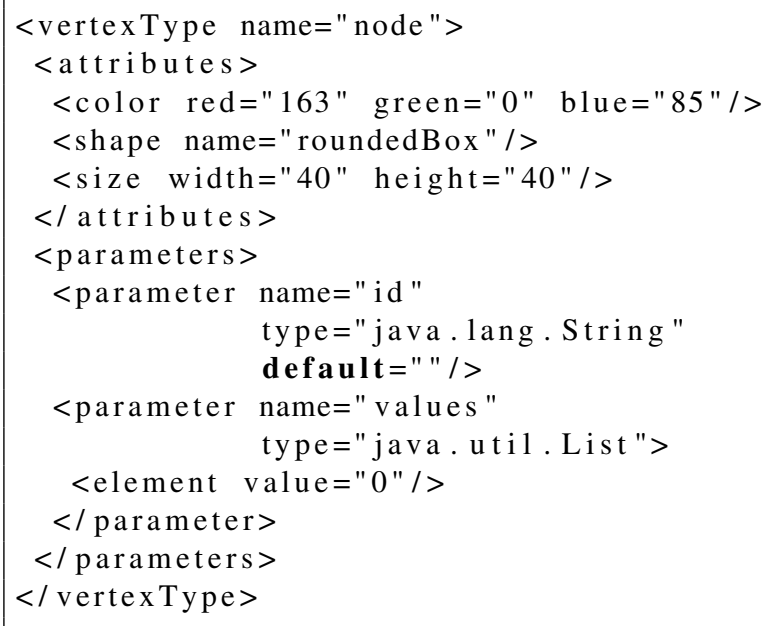

Fig. 4. The type of vertices of the graph shown in Figure 3

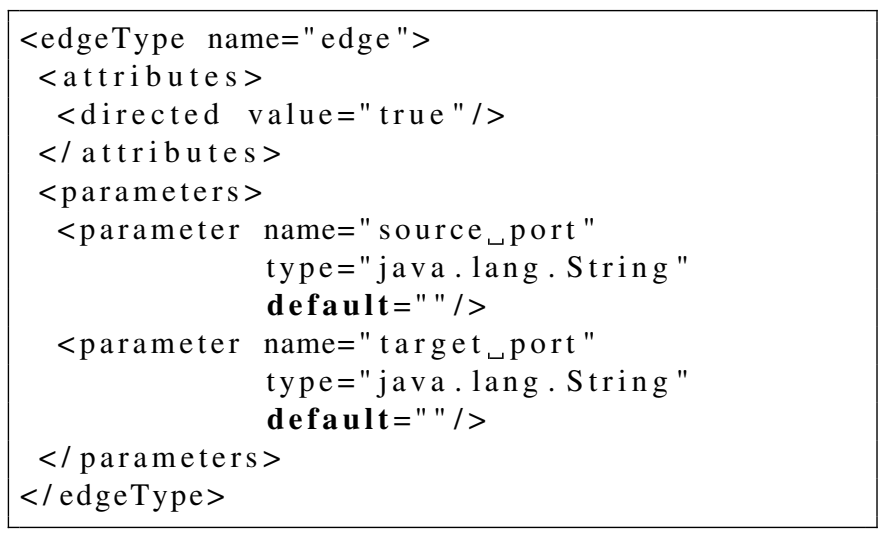

Fig. 5. The type of edges of the graph shown in Figure 3

Graphiti is a stand-alone tool, totally independent of PREESM. However, Graphiti generates workflow graphs, IP-XACT and GraphML files that are the main inputs of PREESM. The GraphML files contain the algorithm model. These inputs are loaded and stored in PREESM by the SDF4J library. This library, discussed in the next section, executes the graph transformations.

\subsection{SDF4J : A Java Library for Algorithm Data Flow Graph Transformations}

SDF4J is a library defining several Data-Flow oriented graph models such as SDF and Directed Acyclic Graph (DAG [33]). It provides the user with several classic SDF transformations such as hierarchy flattening, and SDF to Homogeneous SDF (HSDF [34]) transformations and some clustering algorithms. This library also gives the possibility to expand optimization templates. It defines its own graph representation based on the GraphML standard and provides the associated parser and exporter class. SDF4J is freely available (GPL licence) for download.

\subsubsection{SDF4J SDF Graph model}

An SDF graph is used to simplify the application specifications. It allows the representation of the application behavior at a coarse grain level. This data flow representation models the application operations and specifies the data dependencies between these operations.

An SDF graph is a finite directed, weighted graph $G=<$ $V, E, d, p, c>$ where :

- $V$ is the set of nodes. A node computes an input data stream and outputs the result.

- $E \subseteq V \times V$ is the edge set, representing channels which carry data streams.

- $d: E \rightarrow N \cup\{0\}$ is a function with $d(e)$ the number of initial tokens on an edge $e$. 
- $p: E \rightarrow N$ is a function with $p(e)$ representing the number of data tokens produced at $e$ 's source to be carried by $e$.

- $c: E \rightarrow N$ is a function with $c(e)$ representing the number of data tokens consumed from $e$ by $e$ 's sink node.

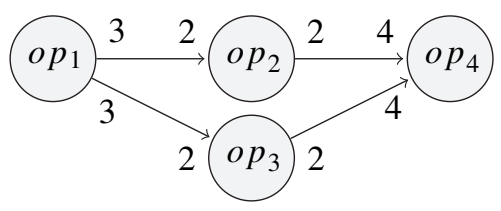

Fig. 6. A SDF graph

This model offers strong compile-time predictability properties, but has limited expressive capability. The SDF implementation enabled by the SDF4J supports the hierarchy defined in [3] which increases the model expressiveness. This specific implementation is straightforward to the programmer and allows user-defined structural optimizations. This model is also intended to lead to a better code generation using common C patterns like loop and function calls. It is highly expandable as the user can associate any properties to the graph components (edge, vertex) to produce a customized model.

\subsubsection{SDF4J SDF Graph transformations}

SDF4J implements several algorithms intended to transform the base model or to optimize the application behavior at different levels.

- The hierarchy flattening transformation aims to flatten the hierarchy (remove hierarchy levels) at the chosen depth in order to later extract as much as possible parallelism from the designer's hierarchical description.

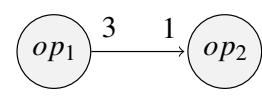

Fig. 7. A SDF graph and its HSDF transformation

- The HSDF transformation (Figure 7) transforms the SDF model to an HSDF model in which the amount of tokens exchanged on edges are homogeneous (production $=$ consumption). This model reveals all the potential parallelism in the application but dramatically increases the amount of vertices in the graph.
- The internalization transformation based on [35] is an efficient clustering method minimizing the number of vertices in the graph without decreasing the potential parallelism in the application.

- The SDF to DAG transformation converts the SDF or HSDF model to the DAG model which is commonly used by scheduling methods [33].

\subsection{PREESM : A Complete Framework for Hardware and Software Codesign}

In the framework, the role of the PREESM tool is to perform the rapid prototyping tasks. Figure 8 depicts an example of a classic workflow which can be executed in the PREESM tool. As seen in Section 3.3, the data flow model chosen to describe applications in PREESM is the SDF model. This model, described in [22], has the great advantage of enabling the formal verification of static schedulability. The typical number of vertices to schedule in PREESM is between one hundred and several thousand. The architecture is described using IPXACT language, an IEEE standard from the SPIRIT consortium [26]. The typical size of an architecture representation in PREESM is between a few cores and several dozen cores. A scenario is defined as a set of parameters and constraints that specify the conditions under which the deployment will run.

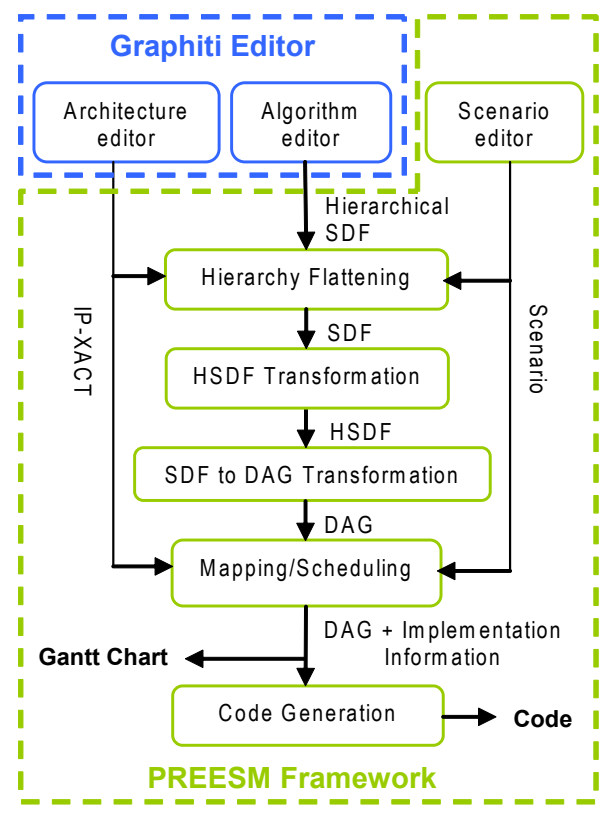

Fig. 8. Example of a workflow graph : From SDF and IP$\mathrm{XACT}$ descriptions to the generated code

As can be seen in Figure 8, prior to entering the scheduling phase, the algorithm goes through three transformation steps : the hierarchy flattening transformation, the HSDF transformation and the DAG transformation (see Section 
3.3.2). These transformations prepare the graph for the static scheduling and are provided by the Graph Transformation Module (see Section 4.1). Subsequently, the DAG - converted SDF graph - is processed by the scheduler [36]. As a result of the deployment by the scheduler, code is generated and a Gantt chart of the execution is displayed. The generated code consists of scheduled function calls, synchronizations and data transfers between cores. The functions themselves are hand-written.

The plug-ins of the PREESM tool implement the rapid prototyping tasks that a user can add to the workflows. These plug-ins are detailed in next section.

\section{THE CURRENT FEATURES OF PREESM}

\subsection{The Graph Transformation Module}

In order to generate an efficient schedule for a given algorithm description, the application defined by the designer must be transformed. The purpose of this transformation is to reveal the potential parallelism of the algorithm and simplify the work of the task scheduler. To provide the user with flexibility while optimizing the design, the entire graph transformation provided by the SDF4J library can be instantiated in a workflow with parameters allowing the user to control each of the three transformations. For example, the hierarchical flattening transformation can be configured to flatten a given number of hierarchy levels (depth) in order to keep some of the user hierarchical construction and to maintain the amount of vertices to schedule at a reasonable level. The HSDF transformation provides the scheduler with a graph of high potential parallelism as all the vertices of the SDF graph are repeated according to the SDF graph's basic repetition vector. Consequently, the number of vertices to schedule is larger than in the original graph. The clustering transformation prepares the algorithm for the scheduling process by grouping vertices according to criteria such as strong connectivity or strong data dependency between vertices. The grouped vertices are then transformed into a hierarchical vertex which is then treated as a single vertex in the scheduling process. This vertex grouping reduces the number of vertices to schedule, speeding up the scheduling process. The user can freely use available transformations in his workflow in order to control the criteria for optimizing the targeted application and architecture.

As can be seen in the workflow displayed in Figure 8, the graph transformation steps are followed by the static scheduling step.

\subsection{The PREESM Static Scheduler}

Scheduling consists of statically distributing the tasks that constitute an application between available cores in a multicore architecture and minimizing parameters such as final latency. This problem has been proven to be NP-complete [37].
A static scheduling algorithm is usually described as a monolithic process, and carries out two distinct functionalities : choosing the core to execute a specific function and evaluating the cost of the generated solutions.

The PREESM scheduler splits these functionalities into three sub-modules [4] which share minimal interfaces : the task scheduling, the edge scheduling and the Architecture Benchmark Computer (ABC) sub-modules. The task scheduling sub-module produces a scheduling solution for the application tasks mapped onto the architecture cores and then queries the $\mathrm{ABC}$ sub-module to evaluate the cost of the proposed solution. The advantage of this approach is that any task scheduling heuristic may be combined with any ABC model, leading to many different scheduling possibilities. For instance, an ABC minimizing the deployment memory or energy consumption can be implemented without modifying the task scheduling heuristics.

The interface offered by the $\mathrm{ABC}$ to the task scheduling sub-module is minimal. The ABC gives the number of available cores, receives a deployment description and returns costs to the task scheduling (infinite if the deployment is impossible). The time keeper calculates and stores timings for the tasks and the transfers when necessary for the ABC.

The ABC needs to schedule the edges in order to calculate the deployment cost. However, it is not designed to make any deployment choices ; this task is delegated to the edge scheduling sub-module. The router in the edge scheduling sub-module finds potential routes between the available cores.

The choice of module structure was motivated by the behavioral commonality of the majority of scheduling algorithms.

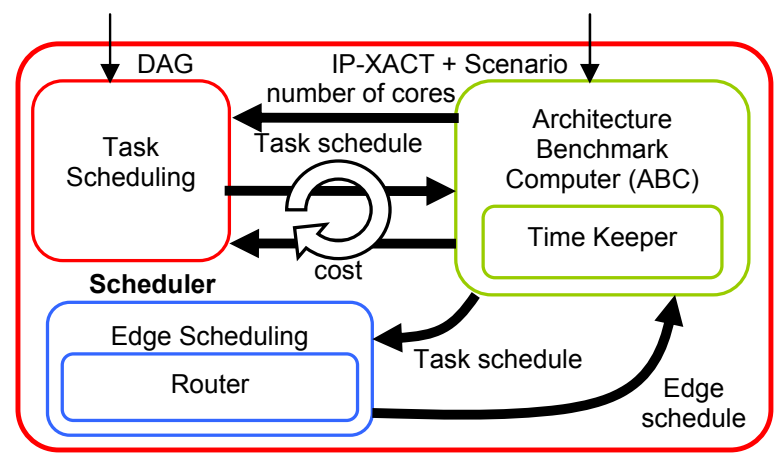

Fig. 9. Scheduler module structure

\subsubsection{Scheduling heuristics}

Three algorithms are currently coded, and are modified versions of the algorithms described in [38].

- A list scheduling algorithm schedules tasks in the order dictated by a list constructed from estimating a critical path. Once a mapping choice has been made, it will 
never be modified. This algorithm is fast but has limitations due to this last property. List scheduling is used as a starting point for other refinement algorithms.

- The FAST algorithm is a refinement of the list scheduling solution which uses probabilistic hops. It changes the mapping choices of randomly chosen tasks; i.e. it associates these tasks to another processing unit. It runs until stopped by the user and keeps the best latency found. The algorithm is multi-threaded to exploit the multi-core parallelism of a host computer.

- A genetic algorithm is coded as a refinement of the FAST algorithm. The $n$ best solutions of FAST are used as the base population for the genetic algorithm. The user can stop the processing at any time while retaining the last best solution. This algorithm is also multithreaded.

The FAST algorithm has been developed to solve complex deployment problems. In the original heuristic, the final order of tasks to schedule, as defined by the list scheduling algorithm, was not modified by the FAST algorithm. The FAST algorithm only modifies the mapping choices of the tasks. In large-scale applications, the initial order of the tasks performed by the list scheduling algorithm becomes occasionally sub-optimal. In the modified version of the FAST scheduling algorithm, the $\mathrm{ABC}$ recalculates the final order of a task when the heuristic maps a task to a new core. The task switcher algorithm used to recalculate the order simply looks for the earliest appropriately sized hole in the core schedule for the mapped task.

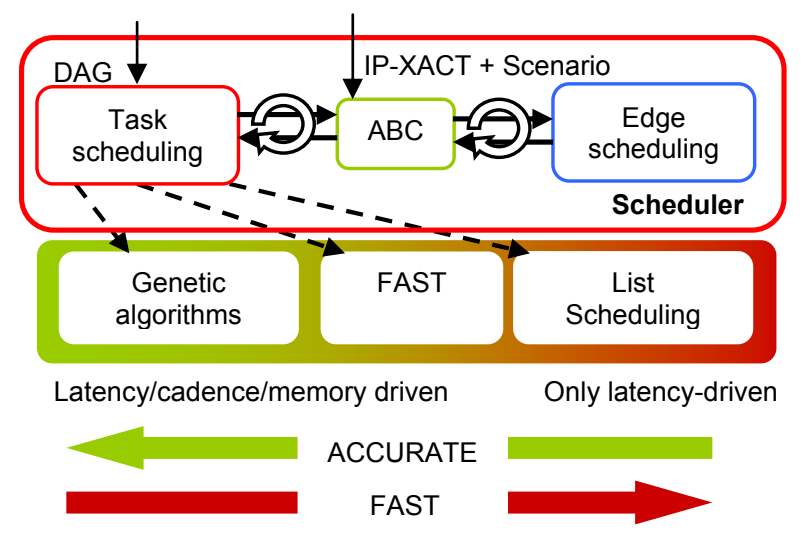

Fig. 10. Switchable scheduling heuristics

\subsubsection{Scheduling architecture model}

The current architecture representation was driven by the need to accurately model multi-core architectures and hardware coprocessors with inter-cores message-passing communication.
This communication is handled in parallel to the computation using Direct Memory Access (DMA) modules. This model is currently used to closely simulate the Texas Instruments TMS320TCI6487 processor (see Section 5.3.2). The model will soon be extended to shared memory communications and more complex interconnections. The term operator represents either a processor core or a hardware coprocessor. Operators are linked by media, each medium representing a bus and the associated DMA. The architectures can be either homogeneous (with all operators and media identical) or heterogeneous. For each medium, the user defines a DMA set-up time and a bus data rate. As shown in Figure 9, the architecture model is only processed in the scheduler by the $\mathrm{ABC}$ and not by the heuristic and edge scheduling sub-modules.

\subsubsection{Architecture Benchmark Computer}

Scheduling often requires much time. Testing intermediate solutions with precision is an especially time-consuming operation. The $\mathrm{ABC}$ sub-module was created by reusing the useful concept of time scalability introduced in SystemC Transaction Level Modeling (TLM) [39]. This language defines several levels of system temporal simulation, from untimed to cycle-accurate precision. This concept motivated the development of several $\mathrm{ABC}$ latency models with different timing precisions. Three ABC latency models are currently coded :

- The loosely-timed model takes into account task and transfer times but no transfer contention.

- The approximately-timed model associates each intercore communication medium with its constant rate and simulates contentions.

- The accurately-timed model adds set-up times which simulate the duration necessary to initialize a parallel transfer controller like Texas Instruments Enhanced Direct Memory Access (EDMA [40]). This set-up time is scheduled in the core which sends the transfer.

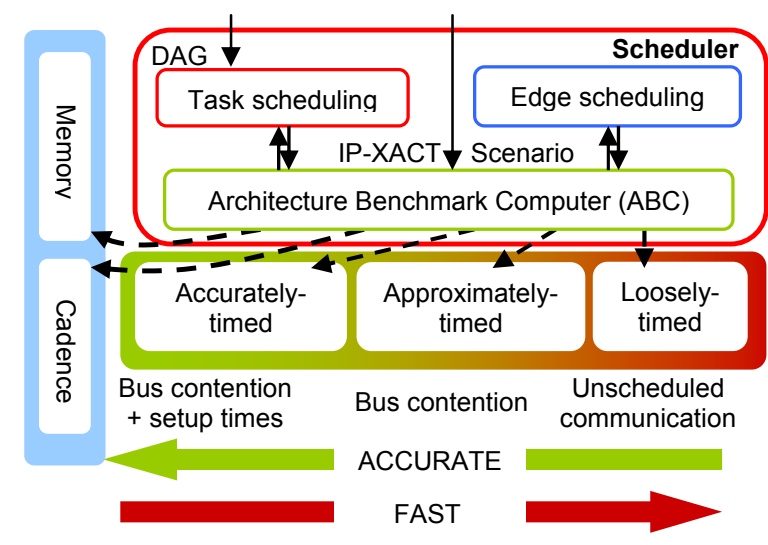

Fig. 11. Switchable ABC models 
The task and architecture properties feeding the ABC submodule are evaluated experimentally, and include media data rate, set-up times and task timings. ABC models evaluating parameters other than latency are planed in order to minimize memory size, memory accesses, cadence (i.e. average runtime), and so on. Currently, only latency is minimized due to the limitations of the list scheduling algorithms : these costs cannot be evaluated on partial deployments.

\subsubsection{Edge scheduling sub-module}

When a data block is transferred from one operator to another, transfer tasks are added and then mapped to the corresponding medium. A route is associated with each edge carrying data from one operator to another, which possibly may go through several other operators. The edge scheduling submodule routes the edges and schedules their route steps. The existing routing process is basic and will be developed further once the architecture model has been extended. Edge scheduling can be executed with different algorithms of varying complexity, which results in another level of scalability. Currently, two algorithms are implemented :

- The simple edge scheduler follows the scheduling order given by the task list provided by the list scheduling algorithm.

- The switching edge scheduler reuses the task switcher algorithm discussed in Section 4.2.1 for edge scheduling. When a new communication edge needs to be scheduled, the algorithm looks for the earliest hole of appropriate size in the medium schedule.

The scheduler framework enables the comparison of different edge scheduling algorithms using the same task scheduling sub-module and architecture model description. The main advantage of the scheduler structure is the independence of scheduling algorithms from cost type and benchmark complexity.

\subsection{Generating code from a static schedule}

Using the AAM methodology from [6], a code can be generated from the static scheduling of the input algorithm on the input architecture (see workflow in Figure 8). This code consists of an initialization phase and a loop endlessly repeating the algorithm graph. From the deployment generated by the scheduler, the code generation module generates a generic representation of the code in XML. The specific code for the target is then obtained after an XSLT transformation. The code generation flow for a Texas Instruments tri-core processor TMS320TCI6487 (see Section 5.3.2) is illustrated by Figure 12.

PREESM currently supports the C64x and C64x+ based processors from Texas Instruments with DSP-BIOS Operating System [41] and the x86 processors with Windows
Operating System. The supported inter-core communication schemes include TCP/IP with sockets, Texas Instruments EDMA3 [42] and RapidIO link [43].

An actor is a task with no hierarchy. A function must be associated with each actor and the prototype of the function must be defined to add the right parameters in the right order. A CORBA Interface Definition Language (IDL) file is associated with each actor in PREESM. An example of an IDL file is shown in Figure 13. This file gives the generic prototypes of the initialization and loop function calls associated with a task. IDL was chosen because it is a language-independent way to express an interface.

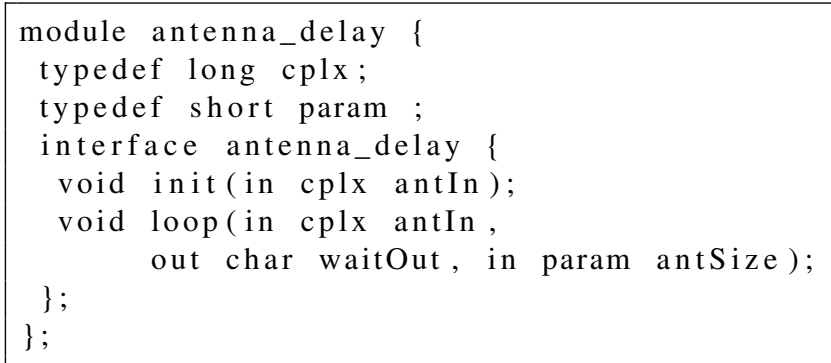

Fig. 13. Example of an IDL prototype

Depending on the type of medium between the operators in the PREESM architecture model, the XSLT transformation generates calls to the appropriate predefined communication library. Specific code libraries have been developed to manage the communications and synchronizations between the target cores [2].

\section{RAPID PROTOTYPING OF A SIGNAL PROCESSING ALGORITHM FROM THE 3GPP LTE STANDARD}

The framework functionalities detailed in the previous sections are now applied to the rapid prototyping of a signal processing application from the 3GPP LTE radio access network physical layer.

\subsection{The 3GPP LTE Standard}

The 3GPP [44] is a group formed by telecommunication organizations to standardize the third generation (3G) mobile phone system specification. This group is currently developing a new standard : the Long Term Evolution (LTE) of the 3G. The aim of this standard is to bring data rates of tens of megabits per second to wireless devices. The communication between the User Equipment (UE) and the evolved base station (eNodeB) starts when the user equipment (UE) requests a connection to the eNodeB via random access preamble (Figure 14). The eNodeB then allocates radio resources to the user for the rest of the random access procedure and sends 


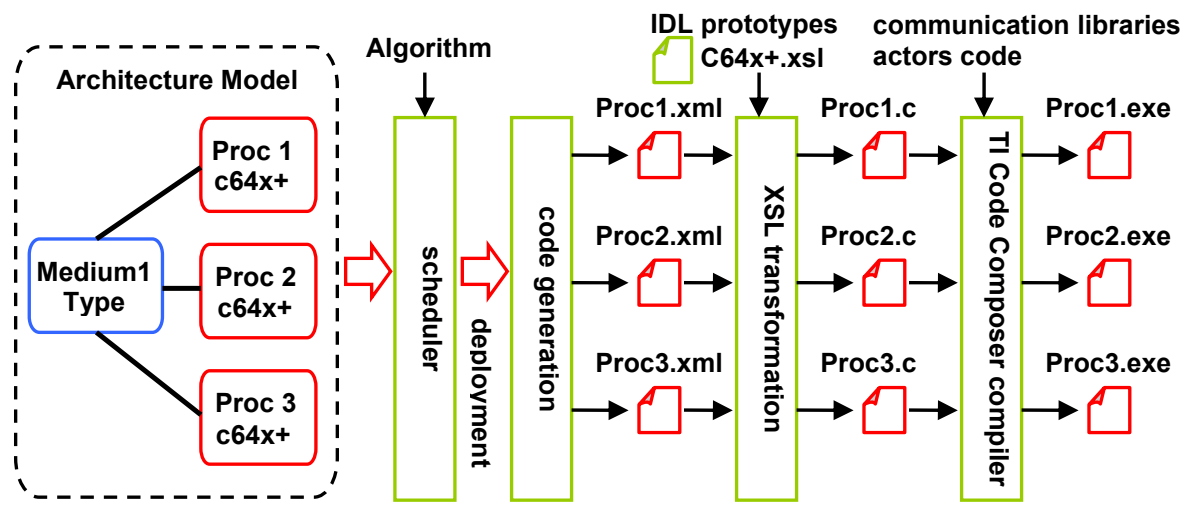

Fig. 12. Code generation

a response. The UE answers with a L2/L3 message containing an identification number. Finally, the eNodeB sends back the identification number of the connected UE. If several UEs sent the same random access preamble at the same time, only one connection is granted and the other UEs will need to send a new random access preamble. After the random access procedure, the eNodeB allocates resources to the UE and uplink and downlink logical channels are created to exchange data continuously. The decoding algorithm, at the eNodeB, of the UE random access preamble is studied in this section. This algorithm is known as the Random Access CHannel Preamble Detection (RACH-PD).

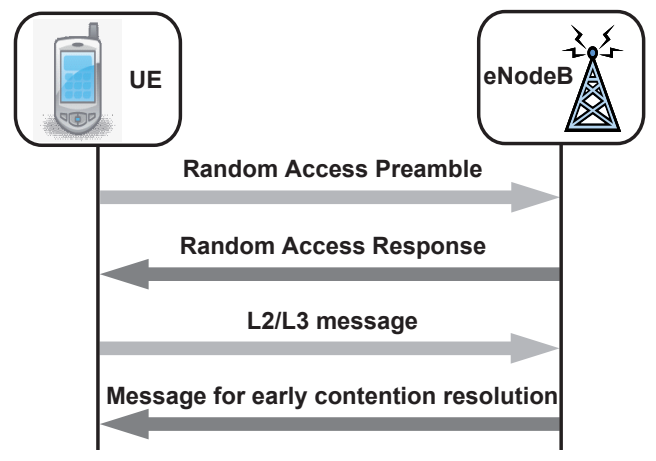

Fig. 14. Random access procedure

\subsection{The RACH Preamble Detection}

The RACH is a contention-based uplink channel used mainly in the initial transmission requests from the UE to the eNodeB for connection to the network. The UE, seeking connection with a base station, sends its signature in a RACH preamble dedicated time and frequency window in accordance with a predefined preamble format. Signatures have special autocorrelation and inter-correlation properties that maximize the ability of the eNodeB to distinguish between different UEs.

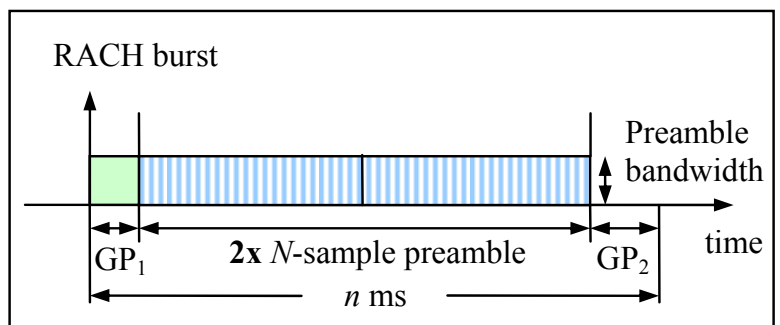

Fig. 15. The random access slot structure

The RACH preamble procedure implemented in the LTE eNodeB can detect and identify each user's signature and is dependent on the cell size and the system bandwidth. Assuming that the eNodeB has the capacity to handle the processing of this RACH preamble detection every millisecond in a worst case scenario.

The preamble is sent over a specified time-frequency resource, denoted as a slot, available with a certain cycle period and a fixed bandwidth. Within each slot, a Guard Period (GP) is reserved at each end to maintain time orthogonality between adjacent slots [45]. This preamble-based random access slot structure is shown in Figure 15.

The case study in this article assumes a RACH-PD for a cell size of $115 \mathrm{~km}$. This is the largest cell size supported by LTE and is also the case requiring the most processing power. According to [46], preamble format\#3 is used with 21,012 complex samples as a cyclic prefix for GP1, followed by a preamble of 24,576 samples followed by the same 24,576 samples repeated. In this case the slot duration is $3 \mathrm{~ms}$ which gives a GP2 of 21,996 samples. As per Figure 16, the algorithm for the RACH preamble detection can be summarized in the following steps [45].

1. After the cyclic prefix removal, the preprocessing (Preproc) function isolates the RACH bandwidth, by shifting the data in frequency and filtering it with downsampling. 


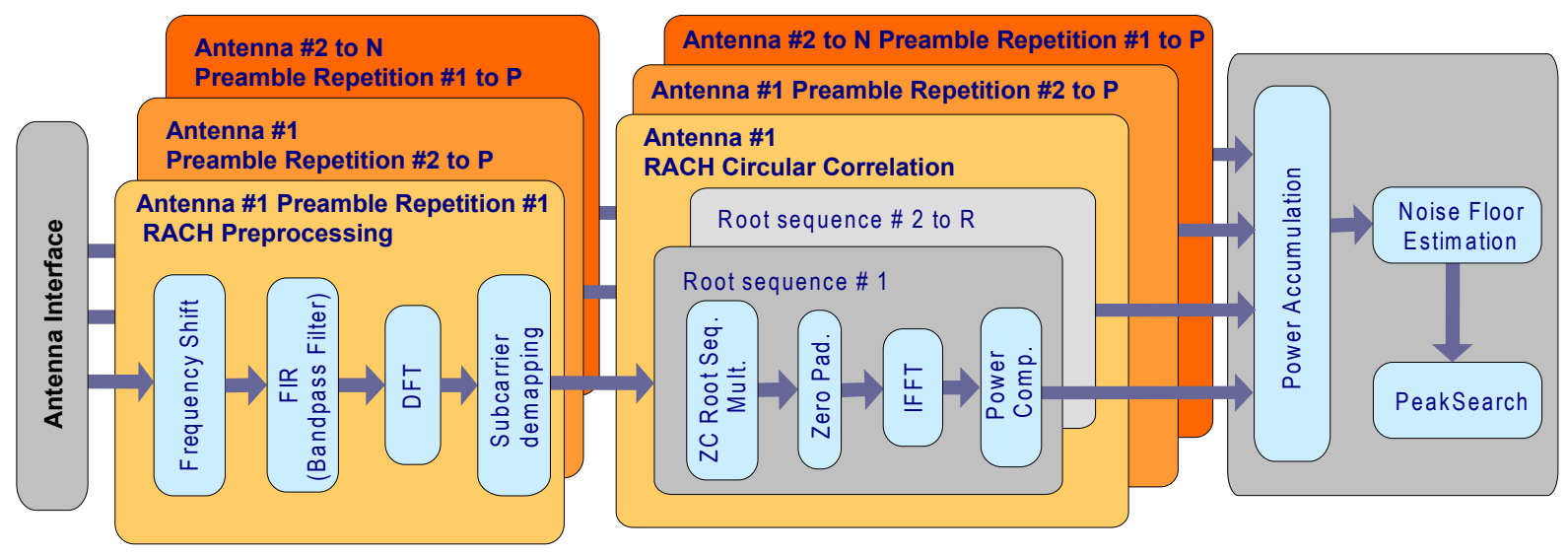

Fig. 16. Random Access Channel Preamble Detection (RACH-PD) Algorithm

It then transforms the data into the frequency domain.

2. Next, the circular correlation (CirCorr) function correlates data with several pre-stored preamble root sequences (or signatures) in order to discriminate between simultaneous messages from several users. It also applies an IFFT to return to the temporal domain and calculates the energy of each root sequence correlation.

3. Then, the noisefloor threshold (NoiseFloorThr) function collects these energies and estimates the noise level for each root sequence.

4. Finally, the peak search (PeakSearch) function detects all signatures sent by the users in the current time window. It additionally evaluates the transmission timing advance corresponding to the approximate user distance.

In general, depending on the cell size, three parameters of RACH may be varied : the number of receive antennas, the number of root sequences and the number of times the same preamble is repeated. The $115 \mathrm{~km}$ cell case implies 4 antennas, 64 root sequences, and 2 repetitions.

\subsection{Architecture Exploration}

\subsubsection{Algorithm Model}

The goal of this exploration is to determine through simulation the architecture best suited to the $115 \mathrm{~km}$ cell RACH-PD algorithm. The RACH-PD algorithm behavior is described as a SDF graph in PREESM. A static deployment enables static memory allocation, so removing the need for runtime memory administration. The algorithm can be easily adapted to different configurations by tuning the HSDF parameters. Using the same approach as in [47], valid scheduling derived from the representation in Figure 16 can be described by the compact expression : (8Preproc) (4(64(InitPower
$(2(($ SingleZCProc $)($ PowAcc $))))$ PowAcc $))$

(64NoiseFloorThreshold)PeakSearch

We can separate the preamble detection algorithm in 4 steps :

1. Preprocessing step : (8Preproc)

2. Circular correlation step : (4(64(InitPower $(2(($ SingleZCProc $)($ PowAcc $))))$ PowAcc $))$

3. Noise floor threshold step : (64NoiseFloorThreshold)

4. Peak search step : PeakSearch

Each of these steps is mapped onto the available cores and will appear in the exploration results detailed in Section 5.3.4. The given description generates 1,357 operations ; this does not include the communication operations necessary in the case of multi-core architectures. Placing these operations by hand onto the different cores would be greatly time-consuming. As seen in Section 4.2 the rapid prototyping PREESM tool offers automatic scheduling, avoiding the problem of manual placement.

\subsubsection{Architecture Exploration}

The four architectures explored are shown in Figure 17. The cores are all homogeneous Texas Instrument TMS320C64x+ Digital Signal Processors (DSP) running at $1 \mathrm{GHz}$ [48]. The connections are made via DMA links. The first architecture is a single-core DSP such as the TMS320TCI6482. The second architecture is dual-core, with each core similar to that of the TMS320TCI6482. The third is a tri-core and is equivalent to the new TMS320TCI6487 [40]. Finally, the fourth architecture is a theoretical architecture for exploration only, as it is a quad-core. The exploration goal is to determine the number of cores required to run the random RACH-PD algorithm in a $115 \mathrm{~km}$ cell and how to best distribute the operations on the given cores. 


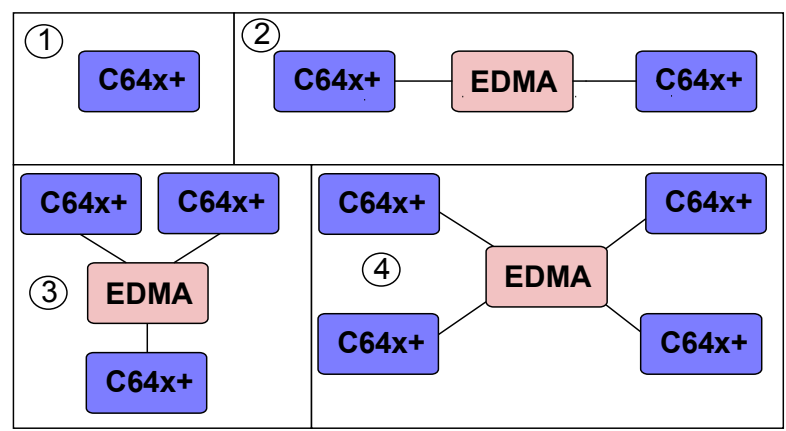

Fig. 17. Four architectures explored

\subsubsection{Architecture Model}

To solve the deployment problem, each operation is assigned an experimental timing (in terms of CPU cycles). These timings are measured with deployments of the actors on a single C64 $\mathrm{x}+$. Since the C64x+ is a 32-bit fixed-point DSP core, the algorithms must be converted from floating-point to fixedpoint prior to these deployments. The EDMA is modelled as a non-blocking medium (see Section 4.2.2) transferring data at a constant rate and with a given set-up time. Assuming the EDMA has the same performance from the L2 internal memory to the L2 internal memory as the EDMA3 of the TMS320TCI6482 (see [42], then the transfer of $\mathrm{N}$ bytes via EDMA should take approximately) : transfer $(N)=$ $135+(N \div 3.375)$ cycles. Consequently, in the PREESM model, the average data rate used for simulation is 3.375 GBytes/s and the EDMA set-up time is 135 cycles.

\subsubsection{Architecture choice}

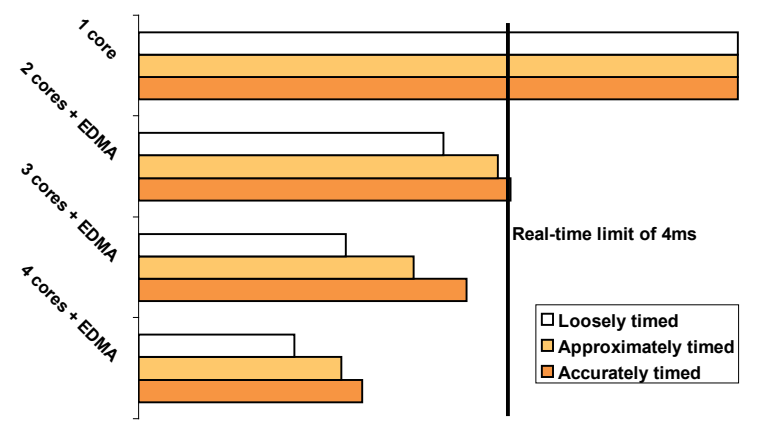

Fig. 18. Timings of the RACH-PD algorithm schedule on target architectures

The PREESM automatic scheduling process is applied for each architecture. The workflow used is close to that of Figure 8. The simulation results obtained are shown in Figure 18. The list scheduling heuristic is used with loosely-timed, approximately-timed and accurately-timed ABCs. Due to the $115 \mathrm{~km}$ cell constraints, preamble detection must be processed in less than $4 \mathrm{~ms}$.

The experimental timings were measured on code executions using a TMS320TCI6487. The timings feeding the simulation are measured in loops, each calling a single function with $\mathrm{L} 1$ cache activated. For more details about $\mathrm{C} 64 \mathrm{x}+$ cache, see [48]. This represents the application behaviour when local data access is ideal and will lead to an optimistic simulation. The RACH application is well suited for a parallel architecture, as the addition of one core reduces the latency dramatically. Two cores can process the algorithm within a time frame close to the real-time deadline with loosely and approximately timed models but high data transfer contention and high number of transfers disqualify it when accurately timed model is used.

The 3-core solution is clearly the best one : its CPU loads (less than $86 \%$ with accurately-timed $\mathrm{ABC}$ ) are satisfactory and do not justify the use of a fourth core, as can be seen in Figure 18. The high data contention in this case study justifies the use of several ABC models; simple models for fast results and more complex models to dimension correctly the system.

\subsection{Code Generation}

Developed Code libraries for the TMS320TCI6487 and automatically generated code created by PREESM (see Section 4.3) were used in this experiment. Details of the code libraries and code optimizations are given in [2]. The architecture of the TMS320TCI6487 is shown in Figure 19. The communication between the cores is performed by copying data with the EDMA3 from one core local L2 memory to another core L2 memory. The cores are synchronized using inter-core interruptions. Two modes are available for memory sharing : in symmetric mode, each CPU has 1MByte of L2 memory while in asymmetric mode, core- 0 has $1.5 \mathrm{Mbyte}$, core- 1 has 1MByte and core-2 0.5MByte.

From the PREESM generated code, the size of the statically allocated buffers are 1.65 Mbytes for one core, 1.25 Mbytes for a second core and 200 kBytes for a third core. The asymetric mode is chosen to fit this memory distribution. As the necessary memory is higher than the internal L2, some buffers are manually chosen to go in the external memory and the $\mathrm{L} 2$ cache [40] is activated. A memory minimization ABC in PREESM would help this process, targeting some memory objectives while mapping the actors on the cores.

Modeling the RACH-PD algorithm in PREESM while varying the architectures (1,2,3 and 4 cores-based) enabled the exploration of multiple solutions under the criterion of meeting the stringent latency requirement. Once the target architecture is chosen, PREESM can be setup to generate a framework code for the simulated solution. As highlighted and explained in the previous paragraph, the statically allocated buffers by the generated code where higher than the physical memory of the target architecture. This necessitated 


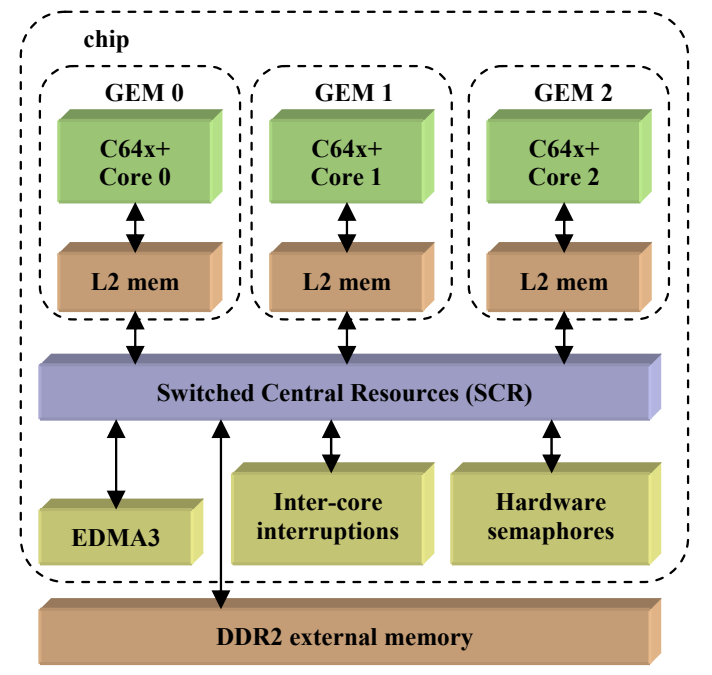

Fig. 19. TMS320TCI6487 architecture

moving manually some of the non critical buffers to external memory. This generated code, representing a priori a good deployment solution, when executed on the target had an average load of $78 \%$ per core while meeting the real time deadline. Hence, the goal of decoding a RACH-PD every $4 \mathrm{~ms}$ on the TMS320TCI6487 is thus successfully accomplished. A simplified view of the code execution is shown in Figure 20. The execution of the generated code had led to a realistic assessment of a deployment very close to that predicted with accurately timed $\mathrm{ABC}$ where the simulation had shown an average load per core around $80 \%$. These results show that prototyping the application with PREESM allows by simulation to assess different solutions and to give the designer a realistic picture of the multi-core solution before solving complex mapping problems. This global result needs to be tempered because one week-effort of manual memory optimizations and also some manual constraints were necessary to obtain such a fast deployment. New ABCs computing the costs of semaphores for synchronizations and the memory balance between the cores will reduce this manual optimizations time.

\section{CONCLUSIONS}

The intent of this paper was to detail the functionalities of a rapid prototyping framework comprising the Graphiti, SDF4J, and PREESM tools. The main features of the framework are the generic graph editor, the graph transformation module, the automatic static scheduler, and the code generator. With this framework, a user can describe and simulate the deployment, choose the most suitable architecture for the algorithm and generate an efficient framework code. The framework has been succesfully tested on RACH-PD algorithm from the 3GPP LTE standard. The RACH-PD algorithm with 1357 operations was deployed on a tri-core DSP and the

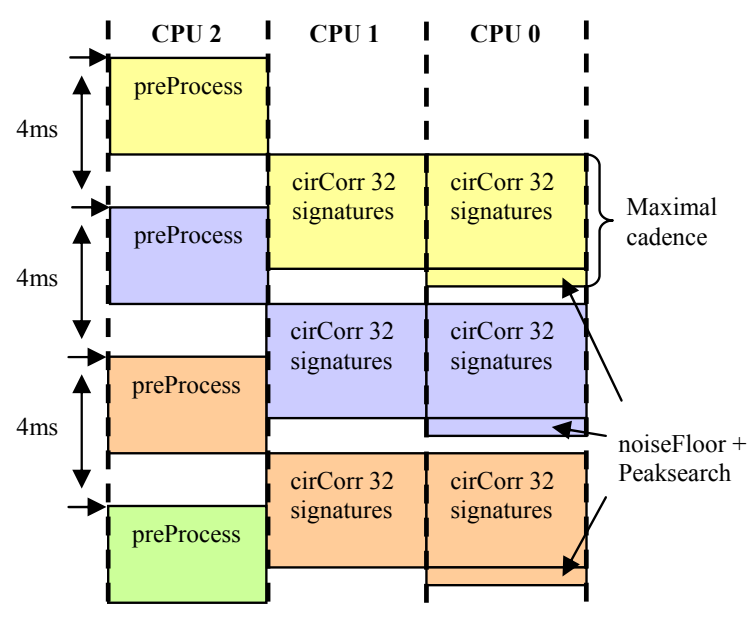

Fig. 20. Execution of the RACH-PD algorithm on a TMS320TCI6487

simulation was validated by the generated code execution. In the near future, an increasing number of CPUs will be available in complex System on Chips. Developing methodologies and tools to efficiently partition code on these architectures is thus an increasingly important objective.

\section{REFERENCES}

[1] E.A. Lee, "The problem with threads," EECS in IEEE Computer, vol. 39, no. 5, pp. 33-42, 2006.

[2] M. Pelcat, S. Aridhi, and J. F. Nezan, "Optimization of automatically generated multi-core code for the LTE RACH-PD algorithm," 0811.0582, Nov. 2008, DASIP 2008, Bruxelles : Belgium.

[3] J. Piat, S. S. Bhattacharyya, M. Pelcat, and M. Raulet, "Multi-core code generation from interface based hierarchy," DASIP 2009, to appear.

[4] M. Pelcat, P. Menuet, S. Aridhi, and J.-F. Nezan, "Scalable compile-time scheduler for multi-core architectures," DATE 2009.

[5] "Eclipse Open Source IDE : Available Online," http://www.eclipse.org/downloads/.

[6] T. Grandpierre and Y. Sorel, "From algorithm and architecture specifications to automatic generation of distributed real-time executives: a seamless flow of graphs transformations," in MEMOCODE '03. Proceedings. First ACM and IEEE International Conference on Formal Methods and Models for Co-Design, 2003, pp. 123132.

[7] “OpenMP," http://openmp.org/wp/. 
[8] R. D. Blumofe, C. F. Joerg, B. C. Kuszmaul, C. E. Leiserson, K. H. Randall, and Y. Zhou, "Cilk: An efficient multithreaded runtime system," JOURNAL OF PARALLEL AND DISTRIBUTED COMPUTING, vol. 37, pp. 207-216, 1995.

[9] “OpenCL," http://www.khronos.org/opencl/.

[10] "The Multicore Association," http://www.multicoreassociation.org/home.php.

[11] "PolyCore Software Poly-Mapper tool," http://www.polycoresoftware.com/products3.php.

[12] E.A. Lee, "Overview of the ptolemy project," Technical memorandum UCB/ERL MO1/11, University of California at Berkeley, 2001.

[13] J. Eker and J. W. Janneck, "CAL Language Report," Tech. Rep., ERL Technical Memo UCB/ERL M03/48, University of California at Berkeley, December 2003.

[14] S. Bhattacharyya, G. Brebner, J. Eker, J. Janneck, M. Mattavelli, C. von Platen, and M. Raulet, "OpenDF - a dataflow toolset for reconfigurable hardware and multicore systems," SIGARCH Comput. Archit. News, 2008.

[15] G. Karsai, J. Sztipanovits, A. Ledeczi, and T. Bapty, "Model-integrated development of embedded software," Proceedings of the IEEE, vol. 91, no. 1, pp. 145-164, 2003.

[16] P. Belanovic, An Open Tool Integration Environment for Efficient Design of Embedded Systems in Wireless Communications, Ph.D. thesis, Technischen Universitat Wien, 2006.

[17] T. Grandpierre, C. Lavarenne, and Y. Sorel, "Optimized rapid prototyping for real-time embedded heterogeneous multiprocessors," in Hardware/Software Codesign, 1999. (CODES '99) Proceedings of the Seventh International Workshop on, 1999, pp. 74-78.

[18] C.-J. Hsu, F. Keceli, M.-Y. Ko, S. Shahparnia, and S. S. Bhattacharyya, "Dif: An interchange format for dataflow-based design tools," 2004.

[19] S. Stuijk, Predictable Mapping of Streaming Applications on Multiprocessors, Ph.D. thesis, Technische Universiteit Eindhoven, 2007.

[20] B.D. Theelen, "A performance analysis tool for Scenario-Aware streaming applications," in Quantitative Evaluation of Systems, 2007. QEST 2007. Fourth International Conference on the, 2007, pp. 269-270.

[21] "Graphiti Editor : Available Online," http://sourceforge.net/projects/graphiti-editor/.
[22] E.A Lee and D.G Messerschmitt, "Synchronous data flow," Proceedings of the IEEE, vol. 75, no. 9, pp. 12351245, sept 1987.

[23] "SDF4J : $\quad$ Available Online," http://sourceforge.net/projects/sdf4j/.

[24] "PREESM : Available Online," http://sourceforge.net/projects/preesm/.

[25] J. W. Janneck, “NL - a Network Language," Tech. Rep., ASTG Technical Memo, Programmable Solutions Group, Xilinx Inc., July 2007.

[26] SPIRIT Schema Working Group, "IP-XACT v1.4: A specification for XML meta-data and tool interfaces," Tech. Rep., The SPIRIT Consortium, March 2008.

[27] U. Brandes, M. Eiglsperger, I. Herman, M. Himsolt, and M. S. Marshall, "Graphml progress report, structural layer proposal," in Graph Drawing - 9th International Symposium, GD 2001 Vienna Austria, P Mutzel, M Junger, and S Leipert, Eds., Heidelberg, 2001, pp. 501-512, Springer Verlag.

[28] J. Piat, M. Raulet, M. Pelcat, P. Mu, and O. Déforges, "An extensible framework for fast prototyping of multiprocessor dataflow applications," in IDT'08: Proceedings of the 3rd International Design and Test Workshop, Monastir, Tunisia, december 2008.

[29] “w3c XML standard," http://www.w3.org/XML/.

[30] “w3c XSLT standard,” http://www.w3.org/Style/XSL/.

[31] "Grammatica parser generator : Available Online," http:/grammatica.percederberg.net/.

[32] J. W. Janneck and R. Esser, "A predicate-based approach to defining visual language syntax," in In Symposium on Visual Languages and Formal Methods, HCC01, Stresa, 2001, pp. 40-47.

[33] J. L. Pino, S. S. Bhattacharyya, and E. A. Lee, "A hierarchical multiprocessor scheduling framework for synchronous dataflow graphs," Laboratory, University of California at Berkeley, 1995.

[34] S. Sriram and S. S. Bhattacharyya, Embedded Multiprocessors: Scheduling and Synchronization, CRC, 1 edition, Mar. 2000.

[35] V. Sarkar, Partitioning and scheduling parallel programs for execution on multiprocessors, Ph.D. thesis, Stanford, CA, USA, 1987.

[36] O. Sinnen and L.A. Sousa, "Communication contention in task scheduling," IEEE Transactions on Parallel and Distributed Systems, vol. 16, no. 6, pp. 503-515, 2005. 
[37] M. R. Garey and D. S. Johnson, Computers and Intractability; A Guide to the Theory of NP-Completeness, W. H. Freeman \and Co., 1990.

[38] Y.-K. Kwok, High-performance algorithms of compiletime scheduling of parallel processors, Ph.D. thesis, Hong Kong University of Science and Technology (People's Republic of China), 1997.

[39] F. Ghenassia, Transaction-Level Modeling with Systemc: Tlm Concepts and Applications for Embedded Systems, Springer-Verlag New York, Inc., 2006.

[40] "TMS320TCI6487 DSP platform, texas instrument product bulletin (SPRT405)," .

[41] "Tms320 dsp/bios users guide (SPRU423F)," .

[42] B. Feng and R. Salman, "TMS320TCI6482 EDMA3 performance, texas instrument technical document (SPRAAG8)," Nov. 2006.

[43] "RapidIO," http://www.rapidio.org/home/.

[44] "The 3rd Generation Partnership Project," http://www.3gpp.org/.

[45] J. Jiang, T. Muharemovic, and P. Bertrand, "Random access preamble detection for long term evolution wireless networks," Patent Nr 20090040918.

[46] "3GPP technical specification group radio access network; evolved universal terrestrial radio access (EUTRA) (Release 8), 3GPP, TS36.211 (V 8.1.0),” .

[47] S.S. Bhattacharyya and E.A. Lee, "Memory management for dataflow programming of multirate signal processing algorithms," Signal Processing, IEEE Transactions on, vol. 42, no. 5, pp. 1190-1201, 1994.

[48] "TMS320C64x/C64x+ DSP CPU and instruction set reference guide, texas instrument technical document (SPRU732G)," Feb. 2008. 